\title{
THE LOCALIZATION OF ECERIFERUM LOCI IN BARLEY. IV. THREE POINT TESTS OF GENES ON CHROMOSOME 7 IN BARLEY
}

\author{
by \\ BODIL SØGAARD \\ Agricultural Research Department - Research Establishment Risø \\ DK-4000 Roskilde \\ and \\ Department of Physiology, Carisberg Laboratory \\ Gamle Carlsbergvej 10 - DK-2500 Copenhagen, Valby
}

Key words: Hordeum vulgare, erectoides, laxatum, variegated, dearistatum, map of ten genes

Eleven three point tests are reported for chromosome 7 in barley. The tests were analysed in the $F_{3}$ generation. The results permit the construction of a map for the ten genes studied.

Earlier work with translocation tester sets and two point tests has shown the following genes to be located on chromosome 7: the eceriferum genes cer- $i$, cer-t, cer-w, cer-zj and cer-zp, the erectoides genes ert-g and ert-n, the laxatum gene lax-a, the variegated gene va and the dearistatum gene ari-c $(4,12,13,14,10,11$, 16). In order to map these genes on the chromosome eleven independent three point tests involving these ten loci have been made.

All mutant markers used are recessive and have generally well expressed morphological characters. Eceriferum denotes organ specific changes in the waxcoating, erectoides dense ears, laxatum lax ears, variegated yellow stripes on leaves, and dearistatum short awns. In each of the eleven three point tests the genes are combined in such a way that all 27 possible $F_{2}$ genotypes can be distinguished by progeny analysis in $F_{3}$. Between 780 and $1759 F_{2}$ plants have been analysed per test cross, and only $F_{2}$ progenies with normal segregation ratios were used for the $F_{3}$ analysis. Environmental influences on crossover frequencies have been minimised by growing the $F_{1}$ generation under standard conditions in the phytotron (2). The resulting map is presented in Figure 1.

\section{MATERIALS AND METHODS}

The following mutants have been used as

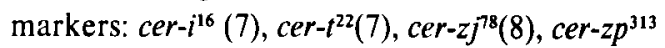
(8), cer-w $w^{48}(8)$, ert-g ${ }^{24}(9), e r t-n^{51}(9)$, lax- $a^{4}(10)$, ari-e $e^{1}(10)$ in Bonus background and $v a(16)$ in Montcalm background. From crosses involving these mutants $(4,12,13,14)$ the following double mutant lines have been isolated;

cer- $t^{22} c e r-z j^{78}$, ari- $e^{1} \quad c e r-z j^{78}, \quad l a x-a^{4}$ cer $-z j^{78}$, cer- $w^{48}$ ert- $\mathrm{g}^{24}$, cer- $t^{22}$ cer-zp $p^{313}$, cer-i $i^{16}$ cer-zp ${ }^{313}$, lax $-a^{4}$ cer-i $i^{16}$, lax $-a^{4}$ ert- $g^{24}$. Each of these double 


\section{B. SøGAARD: Mapping of eceriferum loci in barley IV}

Table II

Observed number of $F_{2}$ plants in 27 genotype classes from the eleven three point tests. Genotypes were determined in $\mathbf{F}_{3}$.

$M=$ wildtype allele; $m=$ the mutant allele which is in coupling with $c$

$\mathrm{C}=$ wildtype allele; $\mathrm{c}=$ the mutant allele which is in coupling with $\mathrm{m}$

$R=$ wildtype allele; $r=$ the mutant allele in repulsion to $c$ and $m$

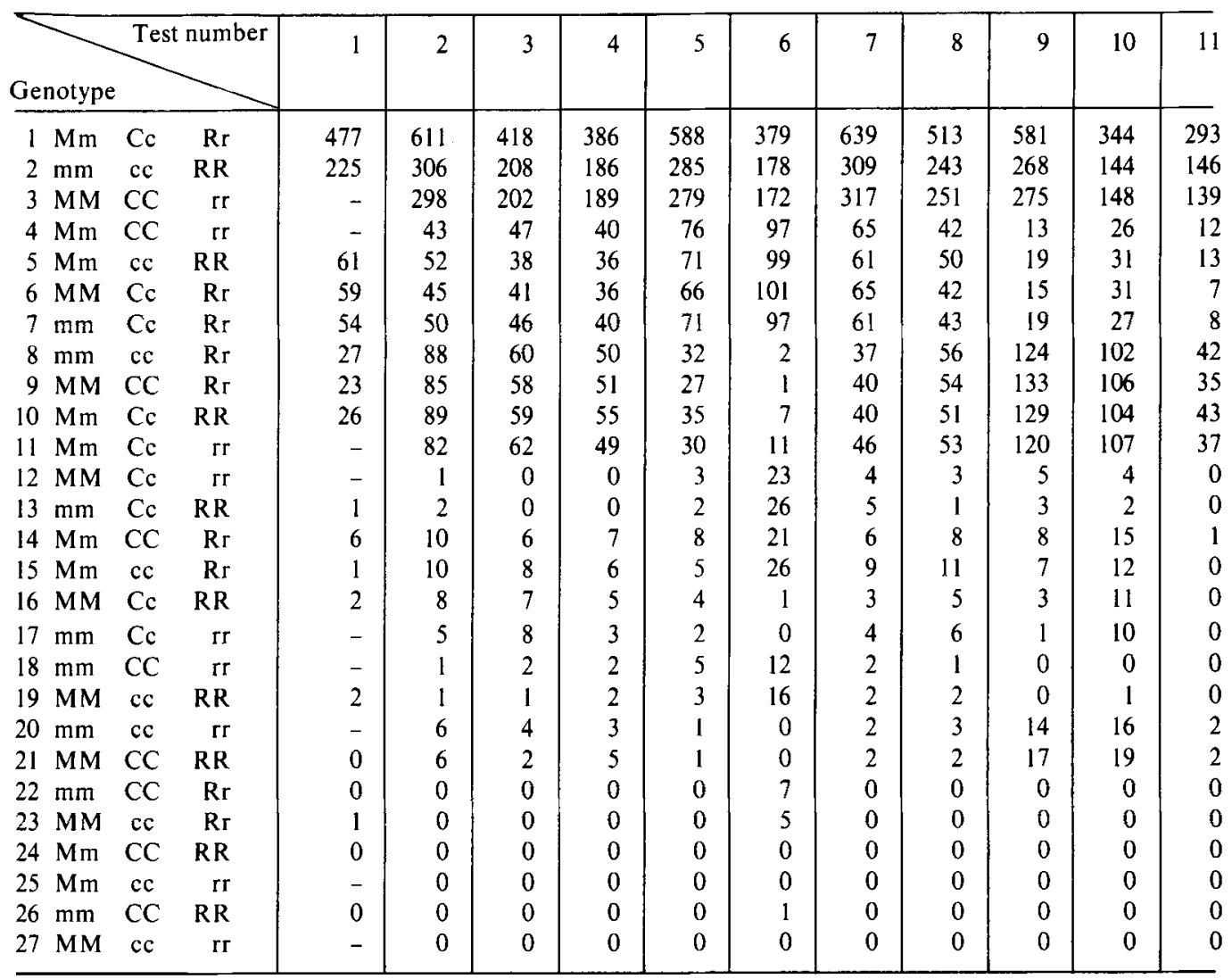

Table III. From this table it can be seen if a particular genotype is the result of fertilization by two non-crossover gametes, by one non-crossover gamete with one crossover gamete, by two crossover gametes and so on. The order of the three loci in each three point test is derived unambigously from Tables II and III. For instance in the tests 1.5 or 7.11 genotypes 12 to 15 are less frequent than genotypes 4 to 7 and 8 to 11 . This implies that the former have arisen by more than one crossover and that therefore marker $c$ lies between marker $m$ and $r$. The derived order of the genes is presented in Figure 2.

The recombination frequencies between the markers as calculated by the maximum likelihood method are given in Figure 2 and the crossover frequencies determined by the approximate method are given in Table IV. The distances as found by the two methods are compared in Table $\mathrm{V}$, where it can be seen that both methods give very similar values. The distance between cer-t and cer-zj has been measured in five different tests to values ranging between 10.5 centimorgan and 12.2 centimorgan. This attests to the uniformity of the present three point tests. Likewise the distance between cer-t and ert-g has been determined three times resulting in distances of $11.3,13.8$ and 14.9 centimorgan. Using the test numbers 5 and 7 a more accurate measure is obtained for this gene distance by adding the recombination frequencies found with the intermediate markers cer-zj and cer-zp respectively. Since the 
Table III

Origin of the genotypes listed in Table II.

$\mathbf{P}=$ parental non-crossover gamete; $\mathbf{I}=$ gamete with crossover in region I; II = gamete with crossover in region II; I + II = double crossover gamete. In test number $1-5,7-11$ corresponds region I to $\mathrm{m}-\mathrm{c}$ and region II to $c-r$, where in test 6 region I corresponds to $m-r$ and region II to c-r.

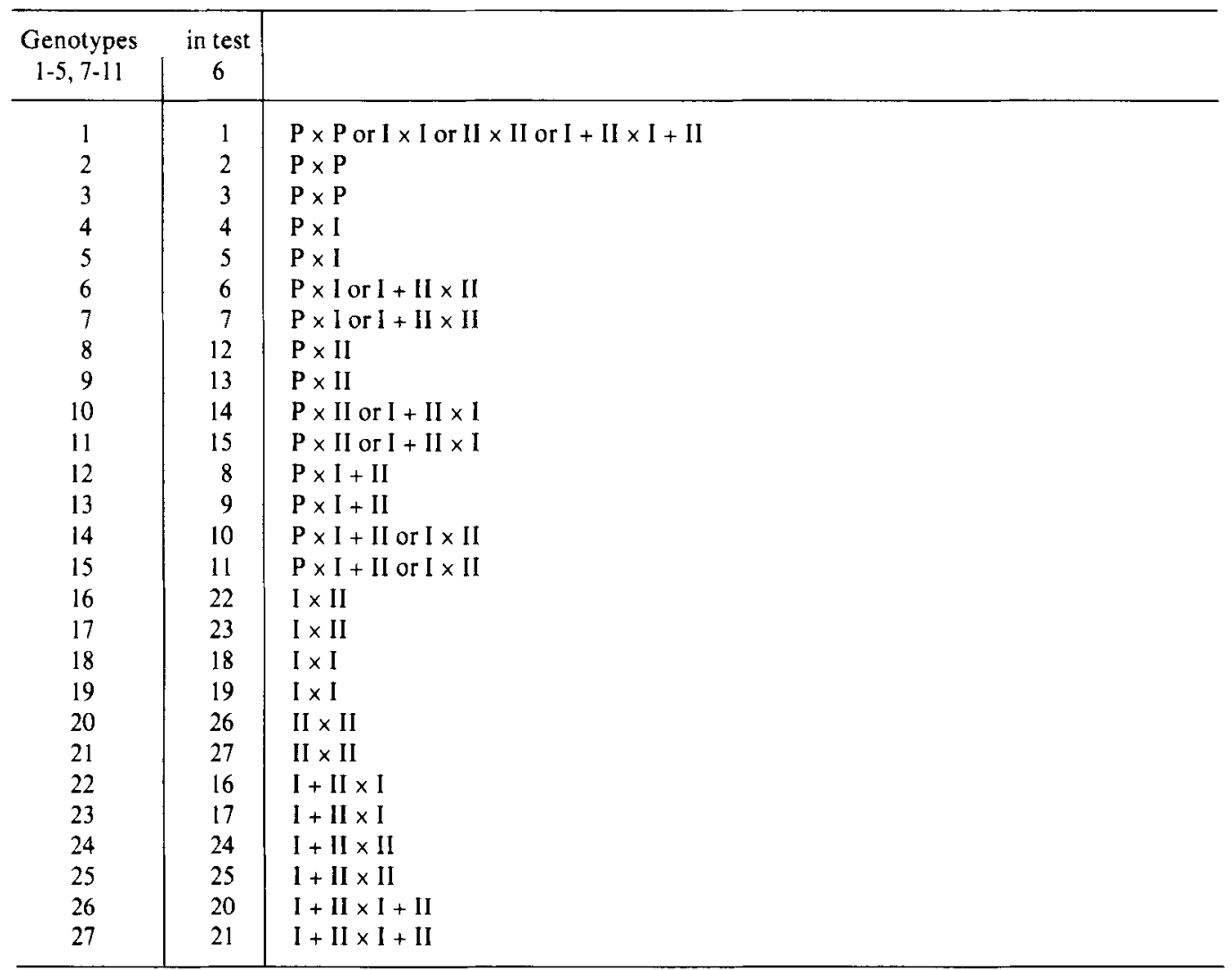

Table IV

Distances obtained from the estimated frequencies for the various genotypes as derived from first principle according to the approximate method (15).

\begin{tabular}{|c|c|c|c|c|c|c|c|c|c|c|c|}
\hline Test number & 1 & 2 & 3 & 4 & 5 & 6 & 7 & 8 & 9 & 10 & 11 \\
\hline \multicolumn{12}{|l|}{ Total number of } \\
\hline Crossovers in & 193 & 9 & 230 & 2 & 100 & 504 & & 2000 & & 520 & 1560 \\
\hline region I & 196 & 234 & 213 & 189 & 340 & 543 & 299 & 223 & 93 & 173 & 41 \\
\hline \multicolumn{12}{|l|}{ Crossovers in } \\
\hline region II & 87 & 428 & 292 & 258 & 156 & 134 & 210 & 268 & 657 & 613 & 174 \\
\hline \multicolumn{12}{|l|}{ Crossovers in } \\
\hline I + II (double) & 4 & 6 & 0 & 0 & 10 & 8 & 18 & 8 & 16 & 12 & \\
\hline Crossover frequency & 10.2 & 6.4 & 8.3 & 8.2 & 10.7 & 21.2 & 8.7 & 7.7 & 2.7 & 6.9 & 2.6 \\
\hline \multicolumn{12}{|l|}{ Crossover frequency } \\
\hline \multicolumn{12}{|l|}{ Double crossovers, } \\
\hline expected & 0.0046 & 0.0076 & 0.0095 & 0.0092 & 0.0052 & 0.0110 & 0.0053 & 0.0072 & 0.0051 & 0.0168 & 0.0029 \\
\hline $\begin{array}{l}\text { Double cro } \\
\text { observed }\end{array}$ & 0.0021 & 0.0017 & 0.0000 & 0.0000 & 0.0031 & $0.003 i$ & 0.0052 & 0.0028 & 0.0046 & 0.0048 & 0.0000 \\
\hline
\end{tabular}


Test number

$\left(F_{1}\right)$

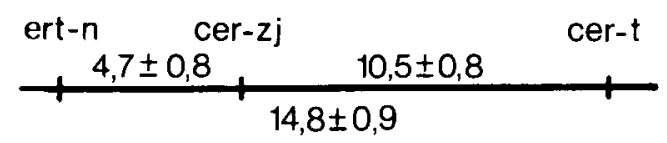

$2(7)$

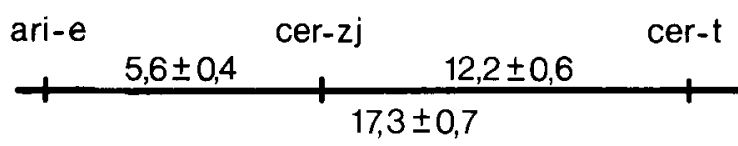

$3(6)$

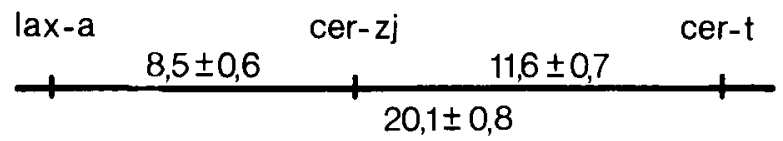

$4(6)$
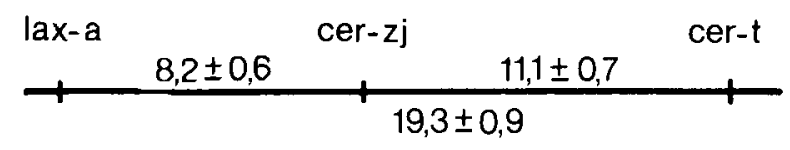

$5(7)$

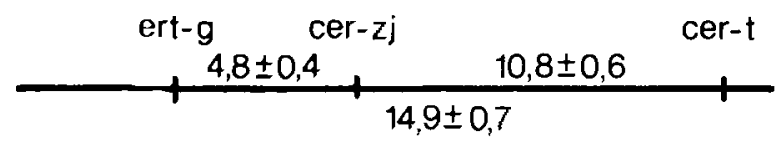

$6(6)$

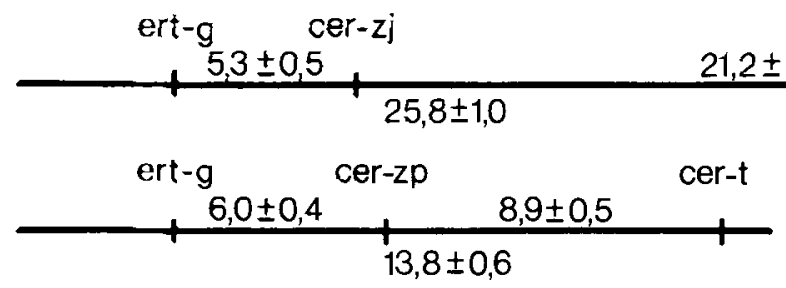

$7(7)$

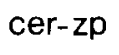

$8(6)$

$9(7)$

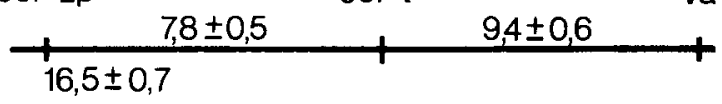

$$
\underbrace{\text { cer-i cer-zp }}_{20,4 \pm 0,8} \quad 18,7 \pm 0,8 \quad \text { va }
$$

$10(6)$

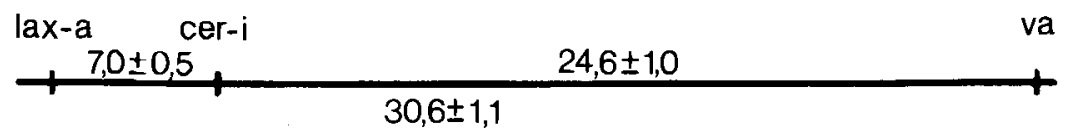

11(4)

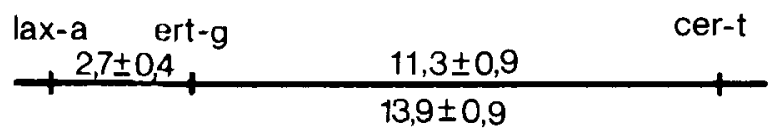

Figure 2. Distances and standard deviations based on the maximum likelihood method of calculation. The numbers in parenthesis designate the number of $F_{1}$ plants i.e. $F_{2}$ families analyzed.

double crossovers are detected with the aid of the intermediate markers the distances increase from 14.9 to 15.6 and from 13.8 to 14.9 centimorgan. The map distance from lax-a to cer-t has been determined in tests $3,4,11$ with crossover frequencies of $201,19.3$ and 13.9 centimorgans. In all three tests no double crossovers were found and the distance thus remains the same if the intermediate marker is used for map distance calculation. 
Table V

Comparison of distances found by two methods of calculation.

\begin{tabular}{c|c|c|c|c}
\hline \multirow{2}{*}{ Test number } & \multicolumn{2}{|c|}{$\begin{array}{c}\text { Region I } \\
\text { Computer } \\
\text { method }\end{array}$} & $\begin{array}{c}\text { Region II } \\
\text { method }\end{array}$ & $\begin{array}{c}\text { Computer } \\
\text { method } \\
\text { method }\end{array}$ \\
\hline 1 & $10.5 \%$ & $10.2 \%$ & $4.7 \%$ & $4.5 \%$ \\
2 & $5.6 \%$ & $6.4 \%$ & $12.2 \%$ & $11.9 \%$ \\
3 & $8.5 \%$ & $8.3 \%$ & $11.6 \%$ & $11.4 \%$ \\
4 & $8.2 \%$ & $8.3 \%$ & $11.1 \%$ & $11.2 \%$ \\
5 & $10.8 \%$ & $10.7 \%$ & $4.8 \%$ & $4.9 \%$ \\
6 & $21.1 \%$ & $21.1 \%$ & $5.3 \%$ & $5.6 \%$ \\
7 & $8.9 \%$ & $8.7 \%$ & $6.0 \%$ & $6.1 \%$ \\
9 & $7.8 \%$ & $7.7 \%$ & $18.7 \%$ & $8.3 \%$ \\
10 & $2.7 \%$ & $2.7 \%$ & $24.6 \%$ & $18.7 \%$ \\
11 & $7.0 \%$ & $6.9 \%$ & $11.3 \%$ & $24.3 \%$ \\
\hline
\end{tabular}

The following distances have been measured twice: cer-zp to cer-t with 7.8 and 8.9 centimorgan; lax-a to cer-zj with 8.5 and 8.2 centimorgan; ert-g to cer-zj with 4.8 and 5.3 centimorgan; cer-zp to $v a$ with 18.7 and 17.2 centimorgan; and cer-i to va with 24.6 and 21.4 centimorgan. Thus, on the whole good reproducibility of individual linkage values has been obtained in this material.

From these linkage values the map in Figure 1 can be constructed. No inconsistencies were met with in arranging the 10 genes in the order given. All map distances of Figure 2 have been weighted with the procedure described by JENSEN and HELMS JøRGENSEN (5). It should however be pointed out that the order of ari-e, ert $g$ and ert-n is not proven by a three point test. This is also the case for the order cer- $i$, $c e r-z j, c e r-z p$ and the order va, cer-w.

Coefficients of coincidence $(\gamma)$ have also been calculated by two methods. One uses the relationship $\mathrm{p}_{3}=\mathrm{p}_{2}-2 \gamma \mathrm{p}_{1} \mathrm{p}_{2}$ in which $\mathrm{p}_{3}$ is the recombination frequency of the two most distant loci, whereas $p_{1}$ and $p_{2}$ are the recombination frequencies in region $\mathrm{I}$ and II respectively. The other method estimates the observed double crossovers from first principles (Table IV) and devides their frequency with the expected number of crossovers. The values obtained are presented in Table VI. No significant differences are apparent between the two types of estimates. In
Table VI

The coefficient of coincidence found in the eleven three point tests.

\begin{tabular}{|c|c|c|}
\hline $\begin{array}{c}\text { Test } \\
\text { number }\end{array}$ & $\begin{array}{l}\text { Coefficient of co } \\
y=\frac{p_{1}+p_{2}-p_{3}}{2 p_{1} p_{2}}\end{array}$ & $\begin{array}{l}\text { idence } \gamma \\
\text { estimation method } \\
\text { from the first } \\
\text { principle }\end{array}$ \\
\hline I & 0.44 & 0.46 \\
\hline 2 & 0.37 & 0.22 \\
\hline 3 & 0.01 & 0.00 \\
\hline 4 & 0.00 & 0.00 \\
\hline 5 & 0.66 & 0.60 \\
\hline 6 & 0.30 & 0.28 \\
\hline 7 & 0.98 & 0.98 \\
\hline 8 & 0.51 & 0.39 \\
\hline 9 & 0.98 & 0,90 \\
\hline 10 & 0.30 & 0.29 \\
\hline 11 & 0.02 & 0.00 \\
\hline
\end{tabular}

all three point tests positive interference is observable. In tests 3,4 and 11 no double crossovers happened to be directly observed which results in coefficients of coincidence of zero. Negative interference as has been found for certain markers of chromosome 1 (15) has not been observed here.

Some of the map distances analysed in the present report have been previously determined by two point tests in $F_{2}$ or $F_{3}$ generations. A summary of these earlier data is compiled in Table VII and compared to the dis- 
Table VII

Recombination frequencies and standard deviations (S.D.) from the present three point tests compared with earlier results from two point tests. Distances in italics were obtained by summation of distances determined with intermediate marker. Data in paranthesis are from materials with abnormal segregation ratios.

\begin{tabular}{|c|c|c|c|c|c|}
\hline & \multirow{2}{*}{$\begin{array}{c}\text { This work } \\
\text { Recombination } \\
\text { frequency and S. D. }\end{array}$} & \multicolumn{4}{|c|}{ Earlier work } \\
\hline & & $\begin{array}{c}\text { Recombination } \\
\text { frequency and S. D. }\end{array}$ & $\begin{array}{c}\text { Number of } \\
\text { plants }\end{array}$ & Generation & Reference \\
\hline lax-a-ert-g & $2.7 \pm 0.4$ & 0 & 460 & $F_{2}$ & (10) \\
\hline lax-a-cer-i & $7.0 \pm 0.5$ & $1.5 \pm 0.5$ & 329 & $F_{3}$ & (14) \\
\hline$l a x-a-c e r-z j$ & $8.4 \pm 0.6$ & $(3.0 \pm 0.6)$ & 369 & $\mathrm{~F}_{3}$ & (14) \\
\hline$l a x-a-c e r-z p$ & $9.4 \pm 0.4$ & $6.0 \pm 1.2$ & 182 & $\mathrm{~F}_{3}$ & (14) \\
\hline ari-e-cer-i $i$ & $4.5 \pm 0.6$ & $5.6 \pm 1.3$ & 152 & $F_{3}$ & (14) \\
\hline ert-g-cer-i & $3.8 \pm 0.4$ & $1.7 \pm 0.7$ & 181 & $\mathrm{~F}_{3}$ & (13) \\
\hline$e r t-g-c e r-z j$ & $5.1 \pm 0.5$ & $5.3 \pm 0.8$ & 399 & $F_{3}$ & (13) \\
\hline ert-g-cer-zp & $6.0 \pm 0.4$ & $7.8 \pm 1.2$ & 263 & $F_{3}$ & (14) \\
\hline ert-g-cer-t & $14.2 \pm 0.6$ & $13.3 \pm 1.9$ & 177 & $\mathrm{~F}_{3}$ & (13) \\
\hline ert-n-cer-zj & $4.7 \pm 0.8$ & $(0.4 \pm 0.3)$ & 234 & $\mathrm{~F}_{3}$ & (14) \\
\hline cer-i-cer-zj & $1.1 \pm 0.4$ & $(4.6 \pm 1.1)$ & 183 & $\mathrm{~F}_{3}$ & (14) \\
\hline$c e r-i-c e r-z p$ & $2.7 \pm 0.3$ & $(4.6 \pm 1.1)$ & 197 & $F_{3}$ & (14) \\
\hline$c e r-i-v a$ & $23.0 \pm 0.6$ & $36.0 \pm 4.0$ & 472 & $F_{3}$ & (13) \\
\hline$c e r-z j-c e r-t$ & $11.2 \pm 0.3$ & $(18.2 \pm 1.8)$ & 286 & $F_{3}$ & (13) \\
\hline cer-zj-va & $20.6 \pm 0.6$ & $(33.0 \pm 4.0)$ & 479 & $F_{2}$ & (13) \\
\hline cer-zj-cer-w & $21.2 \pm 0.9$ & $41.0 \pm 3.0$ & 819 & $F_{2}$ & (14) \\
\hline cer-zp-cer-t & $8.4+0.5$ & $10.0+5.0$ & 446 & $F_{2}$ & (14) \\
\hline$c e r-z p-v a$ & $17.8 \pm 0.8$ & $18.0 \pm 4.0$ & 529 & $F_{2}$ & (14) \\
\hline$c e r-z p-c e r-w$ & $20.5 \pm 1.4$ & $21.0 \pm 3.0$ & 857 & $\mathrm{~F}_{2}$ & (14) \\
\hline$v a-c e r-w$ & $1.5 \pm 1.5$ & $(10.0 \pm 4.0)$ & 540 & $\mathrm{~F}_{2}$ & (14) \\
\hline
\end{tabular}

tances obtained in the three point tests. Of the twenty map distances considered seven give good agreement between the present and the earlier determinations. From the earlier work seven map distances are given in parenthesis, because they include data from $\mathrm{F}_{2}$ families with abnormal segregation ratios and have therefore to be considered less accurate. Abnormal segregation ratios are relatively frequent in barley and can in most cases by traced down to gametic selection. Of the residual six distances those between lax-a to ert-g, cer-i to va, cer-zj to cer- $w$, had been determined by $F_{2}$ analysis only which gives less accurate data for instance due to miss-classification. The large discrepancy between the earlier and present determinations of crossover frequency between lax-a and $c e r-i$ remains unexplained at present. The larger frequency for the lax $a$ to cer- $z p$ distance in the present work as compared to the earlier analysis is at least in part explained by its determination with the aid of an intermediate marker. Likewise the larger distance between ert-g and cer-i in the present work as compared to earlier data probably results from its indirect computation.

It has been mentioned in the Introduction that $\mathrm{F}_{2}$ families with abnormal segregation ratios were not analysed further. In addition the segregation of the individual $F_{2}$ families which were analysed in the $F_{3}$ have been tested by $\boldsymbol{X}^{2}$ analysis for homogeneity before being accepted in the calculations of the crossover frequencies given in Figure 2. This is illustrated in Table VIII for three point test number 2 , which is based on seven $F_{1}$ plants giving the seven $F_{2}$ families 2.1-2.7. Clearly family. 2.7 deviates drastically in crossover frequency both for region 1 (ari-e to cer-zj) and region II (cer-zj to cer-t). This family is therefore rejected. For the $\chi^{2}$-comparison in region I the total crossing over frequency of families 2.1 to 2.6 has been used as expected values (last line in Table VIII). For region II the expected value comprises families 2.1 to 2.6 (last line in Table VIII) as well as all families measuring the crossover frequency be- 
Table VIII

Recombination frequency and $X^{2}$ for three point test number 2.

\begin{tabular}{c|c|r|r|r}
\hline & \multicolumn{2}{|c|}{ Recombination frequency in region } & \multicolumn{2}{|c}{$\chi^{2}$ for region } \\
Test number & I & II & I & II \\
\hline 2.1 & $5.6 \pm 1.0$ & $10.3 \pm 1.4$ & 0.0 & 0.0 \\
2.2 & $5.7 \pm 1.1$ & $9.2 \pm 1.4$ & & \\
2.3 & $6.3 \pm 1.1$ & $12.4 \pm 1.6$ & 0.4 & 1.6 \\
2.4 & $6.1 \pm 1.1$ & $10.9 \pm 1.5$ & 0.2 & 0.1 \\
$2.2+2.5$ & $5.1 \pm 0.7$ & $10.3 \pm 1.0$ & 0.5 & 0.0 \\
2.6 & $5.7 \pm 1.0$ & $11.3 \pm 1.4$ & 0.0 & 0.4 \\
2.7 & $12.7 \pm 1.6$ & $17.8 \pm 1.9$ & 17.0 & 13.3 \\
2 & $5.6 \pm 0.4$ & $12.5 \pm 0.6$ & 0.0 & 4.5 \\
\hline
\end{tabular}

tween cer-zj to cer-t in three point tests $1,3,4$ and 5. The computer program used cannot handle cases, in which the combined recombination frequencies in region $l$ and $l l\left(p_{1}+p_{3}\right)$ are less than the recombination frequencies between the two most distant markers $\left(p_{3}\right)$. This happens to be the case with family 2.5 , which therefore has been combined with the smallest family in the test namely 2.2 . In the other three point tests one family each had to be rejected in test number 8 and 10 because of $x^{2}$-values of 18 , 12 and 13. After these 3 rejections the three point tests were satisfactory to the criteria used by JENSEN \& HELMS JøRGENSEN (5) for consistent mapping.

One way of testing the reliability of the classification is offered by the approximate method applied by SøGAARD (15). The observed frequency distribution of the 27 genotypes in a three point test can be compared with the distribution expected from first principle. An example is given for test number 10 in Table IX with excellent agreement between the two distributions.

Crosses to translocations with a breakage point in the long arm of chromosome 7 give close linkage with most of the eceriferum genes on chromosome $7(4,13,14)$ indicating these genes to be on the long arm. Whereas crossover between cer-t, cer-zj, cer-zp or cer-t and the translocations studied was not observed, more than $10 \%$ crossover occurred in crosses with cer-w (SøGAARD, unpublished). Such large crossover values are believed to indicate a location distally to the translocation breakage point
Table IX

The comparison between the observed and expected phenotype classes in three point test number 10 .

\begin{tabular}{c|c|c}
\hline Genotype & Observed & Expected \\
\hline 1 & 344 & 337.9 \\
2 & 144 & 149.3 \\
3 & 148 & 149.3 \\
4 & 26 & 28.3 \\
5 & 31 & 28.3 \\
6 & 31 & 29.1 \\
7 & 27 & 29.1 \\
8 & 102 & 104.6 \\
9 & 106 & 104.6 \\
10 & 104 & 104.8 \\
11 & 107 & 104.8 \\
12 & 4 & 2.2 \\
13 & 2 & 2.2 \\
14 & 15 & 12.1 \\
15 & 12 & 12.1 \\
16 & 11 & 9.9 \\
17 & 10 & 9.9 \\
18 & 0 & 1.3 \\
19 & 1 & 1.3 \\
20 & 16 & 18.3 \\
21 & 19 & 18.3 \\
22 & 0 & 0.2 \\
23 & 0 & 0.2 \\
24 & 0 & 0.8 \\
25 & 0 & 0.8 \\
26 & 0 & 0.0 \\
27 & 0 & 0.0 \\
\hline
\end{tabular}


(6). This would place the other cer loci into the interstitial segment of the translocations and would orient the linkage map of Figure 1 with lax $-a$ nearest to the centromere.

\section{ACKNOWLEDGEMENTS}

I thank Professor DITER von WETTSTEIN for critical reading of the manuscript and the late Professor OVE Frydenberg and lic. agro. Jens JENSEN for provision of the computer program to calculate the maximum likelihood estimates and for extensive discussion. I am also indebted to the personnel of the Phytotron at the Royal College of Forestry, Stockholm, for growing the plants.

\section{REFERENCES}

1. BAILEY, N.T.J.: Introduction to the mathematical theory of genetic linkage. Clarendon Press, Oxford, pp.1-298 (1961)

2. Dormling, I., A. Gustafsson \& D. von WeTtSTEIN: Phytotron cultivation of Bonus barley: The control of maturation and grain quality. Hereditas 63, 415-428 (1969)

3. Elandt-Johnson, R. C.: Probability models and statistical methods in genetics. John Wiley and Sons, New York, pp. 1-592 (1971)

4. Fester, T. \& B. SøgaARd: The localization of eceriferum loci of barley. Hereditas 61, 327-337 (1969)

5. Jensen, J. \& J. Helms Jørgensen: The barley chromosome 5 linkage map I. Literature survey and map estimation procedure. Hereditas 80 , 5-16 (1975)

6. Kramer, H. H. \& B. A. S. Blander: Orientating linkage maps on the chromosomes of barley. Crop.Sci. 1, 339-342 (1961)

7. LUNDQVIST, U. \& D. von WeTtSTEIN: Induction of eceriferum mutants in barley by ionizing radiation and chemical mutagens. Hereditas 48, 342362 (1962)

8. Lundqvist, U., P. von WettSTEIN-KNowles \& D. von WETTSTEIN: Induction of eceriferum mutants in barley by ionizing radiation and chemical mutagens. Il. Hereditas 59, 473-504 (1968)

9. Persson, G. \& A. Hagberg: Induced variation in quantitative character in barley. Morphology and cytogenetics of erectoides mutants. Hereditas 61 , 115-178 (1968)

10. Persson, G.: An attempt to find suitable genetic markers for dense ear loci in barley 1 . Hereditas 62, 25-96 (1969)

11. Persson, G.: An attempt to find suitable genetic markers for dense ear loci in barley II. Hereditas 63, 1-28 (1969)

12. SøgaARD, B.: The localization of eceriferum loci in barley. II. Cand. scient. thesis, Copenhagen Univ., pp.1-28 (1970)

13. SøgaARD, B.: Linkage studies on eceriferum mutants in barley. Barley Genetic Newsletter 1, 41-47 (1971)

14. SøgaArd, B.: Continued linkage studies on eceriferum mutants in barly. Barley Genetics Newsletter 3, 57-61 (1973)

15. SøgaARD, B.: The localization of eceriferum loci in barley. III. Three-point tests of genes on chromosome 1 in barley. Hereditas $41,41-48$ (1974)

16. Walker, G. W. R., J. Dietrich, R. Miller \& K. KASHA: Recent barley mutants and their linkages. II. Genetic data for further mutants. Can. J. Genet. Cytol. 5, 200-219 (1963) 